\title{
The use of Liquid Crystal Thermography and Particle Image Velocimetry in the Exploration of Heat Transfer Measurements
}

\author{
J. Stasiek ${ }^{1}$, A. Stasiek ${ }^{1}$, D. Mikielewicz ${ }^{1}$, S. Blonski ${ }^{2}$, T.A. Kowalewski ${ }^{2}$ \\ ${ }^{1}$ Gdansk University of Technology, Department of Heat Technology, \\ ul. Narutowicza 11/12,80-233 Gdansk, Poland, jstasiek@pg.gda.pl \\ ${ }^{2}$ IPPT PAN, Polish Academy of Sciences, \\ ul. Pawińskiego 5B, 02-106 Warszawa,Poland, tkowale@ippt.gov.pl
}

\begin{abstract}
Generation of vortices enhances heat transfer by swirl, flow destabilization and development of viscous layers. They may increase heat transfer by several hundred percent. Prior to the use of vortices to influence heat transfer it must be known how different vortices are generated and controlled, and how they interact with the base flow and temperature field. To select the most appropriate vortex generators (VG) for a given task it is necessary to know the heat transfer and flow losses associated with the generation of a specific vortex system. The aim of the paper is to asses the state of art and to encourage exploration of heat transfer control by vortices. Flow visualization and heat transfer experiments were conducted using an open low-speed wind tunnel equipped with Liquid Crystals Thermography (LCT) and Particle Image Velocimetry (PIV). In this work we consider five types of transverse vortex generators (TVGs) which where brought to required temperatures by the hot film method. Heat transfer measurements were carried out by LCT.
\end{abstract}

\section{Introduction}

Three passive mechanisms exist to increase convective heat transfer: (1) swirl, (2) flow destabilization, and (3) developing viscous layers. Vortices and their generators incorporate all three enhancement mechanisms. Vortices swirl fluid around their axis of rotation, they induce velocity profiles which are less stable, and their generation implies flow separation and developing viscous layers [2,3]. Even though everybody knows what is meant by a vortex, namely swirling flow, a clear mathematical definition of a vortex is missing, even though vorticity $\nabla \times \varpi$, and also vortex line and vortex tube are well defined. That vortices do not only swirl but also destabilize the flow is well known for transverse vortices (TV), the Karman vortex street behind a cylinder in cross flow is probably the best known example. The destabilization effects of longitudinal vortices (LV) are still under investigation. One of the reasons is that single LVs are more difficult to generate than TVs. Self-sustained oscillations associated with vortices have been exploited very little in general, especially for heat transfer purposes. The third mechanism viscous layer interruption and initiation of new developing viscous layers is implied by vortex generation [3]. Vortices are generated by fluid friction and its separation, thus vortex generator (VG) surfaces induce development of a new viscous layers. In addition vortices have two properties which make them even more attractive for heat transfer manipulations. They have the tendency to last long and to have small cores which allow to influence heat transfer over long and narrow regions. Both properties follow from Helmholtz vortex theorem (1858) and its extension to viscous flows. In viscous flow vortices can diffuse but cannot start or end in the flow field and therefore have to be generated at solid boundaries by separation. 
To use vortices to influence heat transfer it must be found how different vortices are generated and controlled and how they interact with the original or base flow and temperature field. Special attention will be given to the influence of geometrical VG variations on selfsustained oscillations, flow visualization, and heat transfer augmentation.

In this paper, measurements of local heat transfer coefficients (Nusselt numbers) in a ribroughened rectangular channel (aspect ratio $A R=6.375$, hydraulic diameter $D_{h}=0,06911 \mathrm{~m}$ ) are presented. Many parameters affect the performance of rib-roughened channels: the shape and dimensions of the channel (including constant and variable cross section channels and multiple sharp turns), the rib characteristics (including shape, size, location and thermal conductivity), the convective flow characteristics (through the Reynolds and Prandtl numbers) and the rotation of the blade. The most important parameters were found to be the rib pitch-toheight ratio $\mathrm{P} / \delta$, the rib height-to-hydraulic diameter ratio $\delta / \mathrm{D}$, the aspect ratio (AR) of the rectangular channel (defined as the ratio between the width $\mathrm{W}$ of the ribbed side and the channel height $\mathrm{H}$ ) and the Reynolds number Re.

Liquid crystals were used to determine the distribution of the surface temperature, and then for evaluation of the heat transfer coefficient or the Nusselt number. The flow pattern produced by transverse vortex generators was visualized using a planar beam of double-pulse laser tailored by a cylindrical lens. Sequential images of seeding particles in a cross sectional plane taken with a CCD video camera from the downstream side the flow were stored on a personal computer to obtain distributions of velocity vectors by means of the PIV method.

\section{Liquid Crystal Thermography}

Thermochromic liquid crystals (TLC) and true-colour digital image processing have been successfully used in nonintrusive technical, industrial and biomedical studies and applications. Thin coatings of TLC's at surfaces are utilized to obtain detailed temperature distributions and heat transfer rates for steady or transient processes.

Liquid Crystals Thermography have been extensively applied to the qualitative visualization of entire steady state, or transient temperature fields on solid surfaces. Since quantifying colour is a difficult and somewhat ambiguous task, application of thermochromic liquid crystals initially was largely qualitative. Application of the colour films or interference filters was tedious and inaccurate. The first application of true-colour digital image processing gave impact to qualitative and fast temperature measurements [6]. Rapid development of image processing techniques has made it now possible to set-up inexpensive systems capable of real-time transient full field temperature measurements using TLCs.

Before the execution of a thermal or flow visualization experiment, we should consider the characteristics of the overall relation of the TLC illumination angle, location of the light source, the camera, and make a rational plan for the total measurement system. The relationship between the temperature of the crystal and the measured Hue of the reflected light defines the calibration curve for the specified liquid crystal [8]. The distribution of the colour component pattern on the liquid crystal layer was measured by RGB colour camera and a series of images at different temperature defines the calibration. Two main methods of surface temperature measurements are performed involving steady state and transient techniques. A brief history of these is given by Baughn et al [1] and Stasiek [8]. 


\subsection{Steady State Analyses - Constant Flux Method}

The steady state techniques employ an electrically heated model and the TLC is used to monitor the surface temperature $\mathrm{T}_{\mathrm{w}}$, it gives the local heat transfer coefficient, $\mathrm{h}$ :

$$
q=I^{2} r \text { and } h=\frac{q}{T_{w}-T_{a}}
$$

where $T_{a}$ is driving gas temperature, $I$ is the electrical current and $r$ is the electrical resistance of the heater [8].

\subsection{Steady State Analyses - Uniform Temperature Method}

The TLC-coated test specimen forms one side of a constant temperature water bath and is exposed to a cold/hot air flow. In this case, the heat transfer coefficient, h:

$$
h\left(T_{a}-T_{w}\right)=\frac{k}{x}\left(T_{w}-T_{b}\right)
$$

where $T_{b}$ is a water-side temperature of the wall, $x$ the wall thickness and $k$ the thermal conductivity.

\subsection{Transient Method}

This technique requires measurement of the elapsed time to increase the surface temperature of the TLC coated test specimen from a known predetermined value of initial temperature. Leiner et al [5] developed the formula for evaluation of heat transfer coefficient $h$ :

$$
h=-\frac{\delta \rho c}{t} \ln \left[\frac{T_{a}-T}{T_{a}-T_{i}}\right]
$$

where $\delta$ is a plate wall thickness. The transient local surface temperature $\mathrm{T}$ is detected after a time interval $t, \rho$ and $c$ are the model density and specific heat, $T_{i}$ and $T_{a}$ are the initial wall and gas temperatures and $\mathrm{t}$ is time from initiation of the flow.

\subsection{Experimental Apparatus}

The experimental study was carried out using an open low-speed wind tunnel consisting of entrance section with fan, heaters, large settling chambers with diffusing screen, honeycomb, and working sections as shown in Fig. 1. Air is drawn through the tunnel using a fan able to obtain flow Reynolds number between 500 and 40 000. The working air temperature in the rig ranges between $15^{\circ} \mathrm{C}$ to $65^{\circ} \mathrm{C}$ produced by the heater or cooler positioned just downstream of the inlet. The major construction material of the wind tunnel is perspex. Local and mean velocity are measured using conventional Pitot tubes and DISA hot-wire velocity probe. 


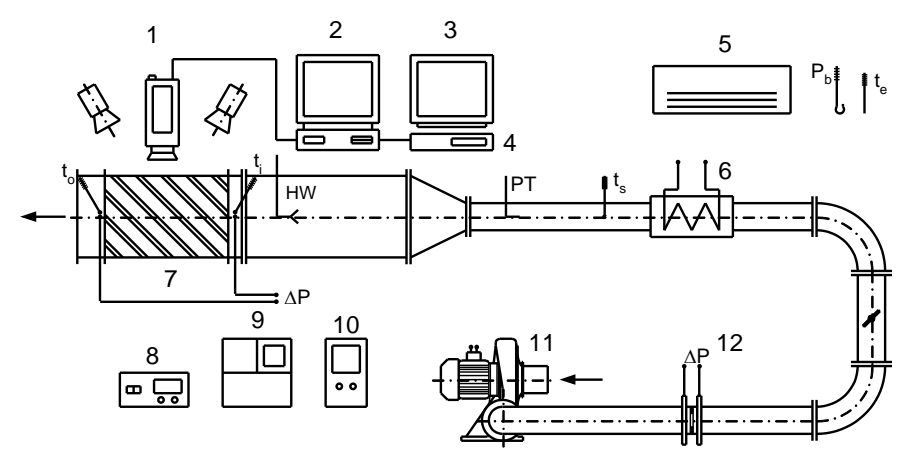

Figure 1: Open low speed wind-tunnel, 1 - RGB camera (TK-1070), 2 - PC, 3 - monitor (RGBVMR 200), 4 - S-VHS recorder, 5 - air conditioning system, 6 - heater, 7 - LC mapping section, 8 digital micro manometer FCO 12, 9 - DISA hot wire system, 10 - variac, 11 - fan, 12 - orifice.
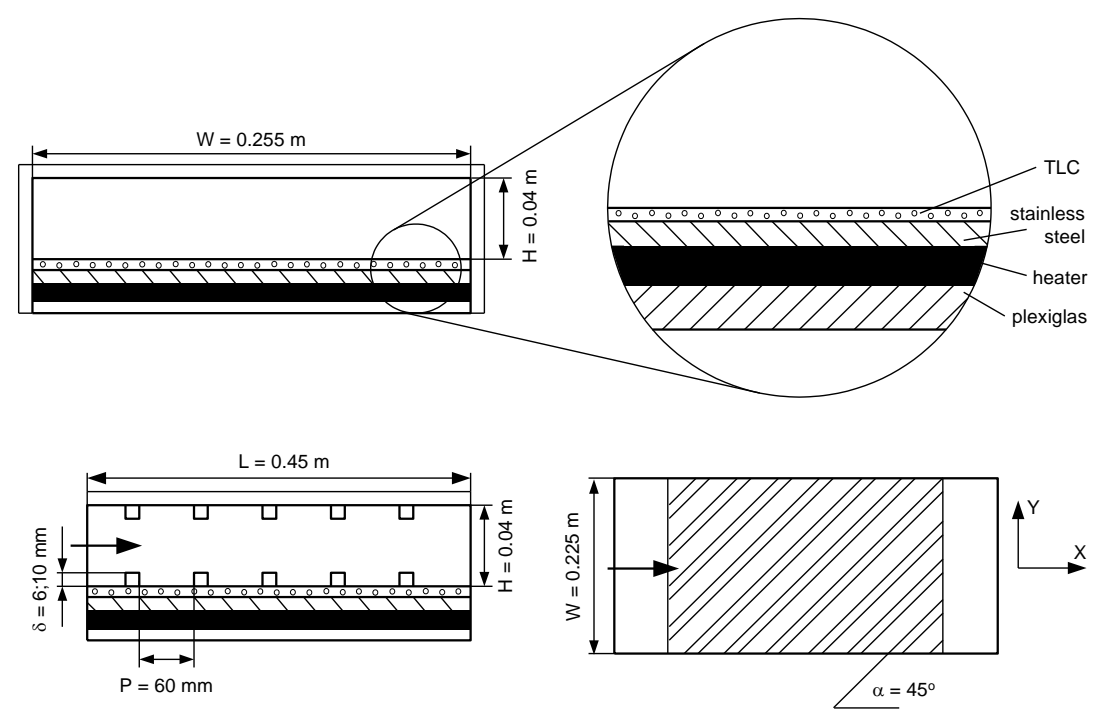

Figure 2: Schematic diagram of the rib roughened test surfaces geometry

Liquid Crystal Tomography and Particle Image Velocimetry measurements were performed in a flat transparent test section with unique rib-corrugation of the walls (Figs. 2, 3 and 4). Ribs were a square $6 \mathrm{~mm}$ rods, placed on the top or bottom (or both) test section wall. In all cases distances between ribs were $60 \mathrm{~mm}$ (distance between edges of the neighbour ribs, measured in the flow direction). In this paper we consider five different configuration of the wall corrugation (Fig. 3):

- first configuration: ribbed bottom wall only by 6 ribs placed perpendicularly to the flow direction (Fig. 3a),

- second configuration: ribbed top and bottom walls, each by 6 ribs placed perpendicularly to the flow direction (Fig. 3b),

- third configuration: ribbed bottom wall only by 6 ribs; ribs were inclined at $45^{\circ}$ to the flow direction (Fig. 3c),

- fourth configuration: ribbed top and bottom walls, 6 ribs on each wall, ribs parallel to each other (ribs on the top and bottom walls) and inclined at $45^{\circ}$ to the flow direction (Fig. 3d),

- fifth configuration: ribbed top and bottom walls, 6 ribs on each wall, ribs perpendicular to each other (ribs on the top and bottom walls) an inclined at $45^{\circ}$ to the flow direction (Fig. 3e). 

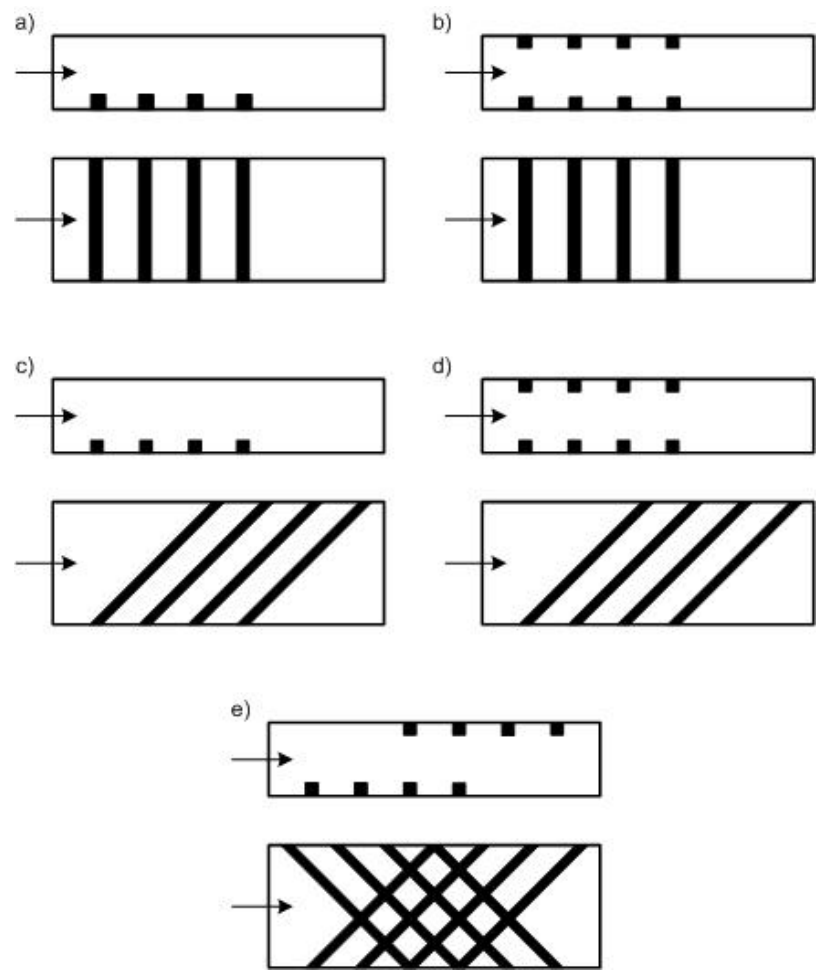

Figure 3: Schematic, top and side views of five types of transverse vortex generators (crosscorrugated and rectangular ribs).

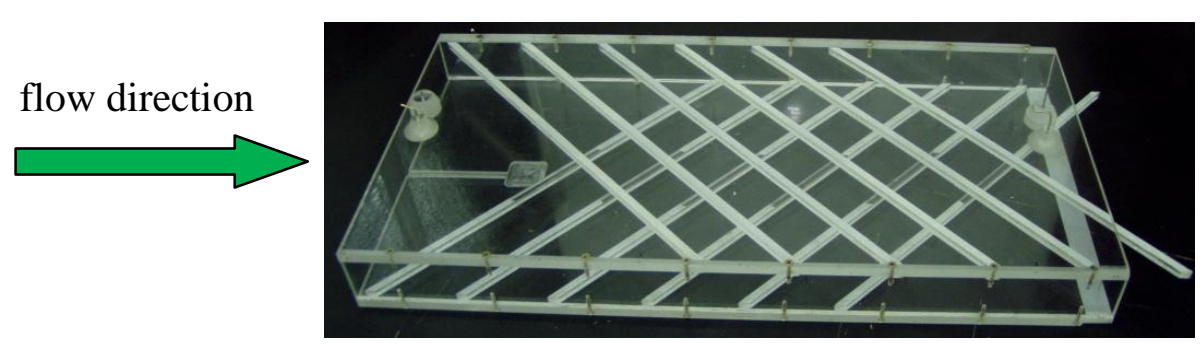

Figure 4. Photo of the test section; configuration with ribbed top and bottom walls; ribs are perpendicular to each other (ribs on the top and bottom walls) and inclined at $45^{\circ}$ to the flow direction (comp. Fig. 3e).

The alternative effects of constant wall temperature and constant heat flux boundary conditions are obtained using electric heater. The TLC mapping section or rib-roughened passages was a rectangular channel as wide and high as the entry and exit sections and delimited by a thin heated plate (width $\mathrm{W}=0.255 \mathrm{~m}$ and length $\mathrm{L}=0.45 \mathrm{~m}$ ) and lateral and frontal unheated Plexiglas walls. The plate was made of $0.5 \mathrm{~mm}$ thick stainless steel to which a plane heater [11] had been attached to provide a controllable uniform heat flux. A thin TLC sheet was applied on the side opposite to the heater to measure local wall temperature. The pre-packaged TLC sheet $(0.15 \mathrm{~mm}$ thick) consisted of a thermochromic liquid layer on a black background applied onto a Mylar film and backed with a pressure-sensitive adhesive. For a given wall temperature, the corresponding colour displayed by the TLCs was digitized and processed in order to obtain, pixel by pixel, the hue, intensity and saturation values (HIS). 
Among three evaluated parameters (HIS) only the hue was retained since it was found to have unique correlation with the surface temperature. Photographs were taken using a RGB videocamera and a true-colour image processing technique. The liquid crystals used here were manufactured in sheet form by Merck Ltd [10] had an event temperature range of 30,0 $35,0^{\circ} \mathrm{C}$. In this particular experiment uncertainty was estimated as about $\pm 0.05^{\circ} \mathrm{C}$ by considering only the section of the surface used in the experiment, span-wise non-uniformities in Hue value are minimized.

\section{Particle Image Velocimetry (PIV)}

Particle Image Velocimetry technique is a well-established experimental method in fluid mechanics, that allows quantitative measurement of two-dimensional flow structure. It enables the measurement of the instantaneous in-plane velocity vector field within a planar section of the flow field and allows to calculate spatial gradients, dissipation of turbulent energy, spatial correlations, and the like [4,7]. In PIV technique selected cross-section of the investigated seeded flow is illuminated by laser light formed in thin "light sheet" (Fig.5). Images of the flow are recorded by CCD camera and correlated to calculate instantaneous velocity fields.

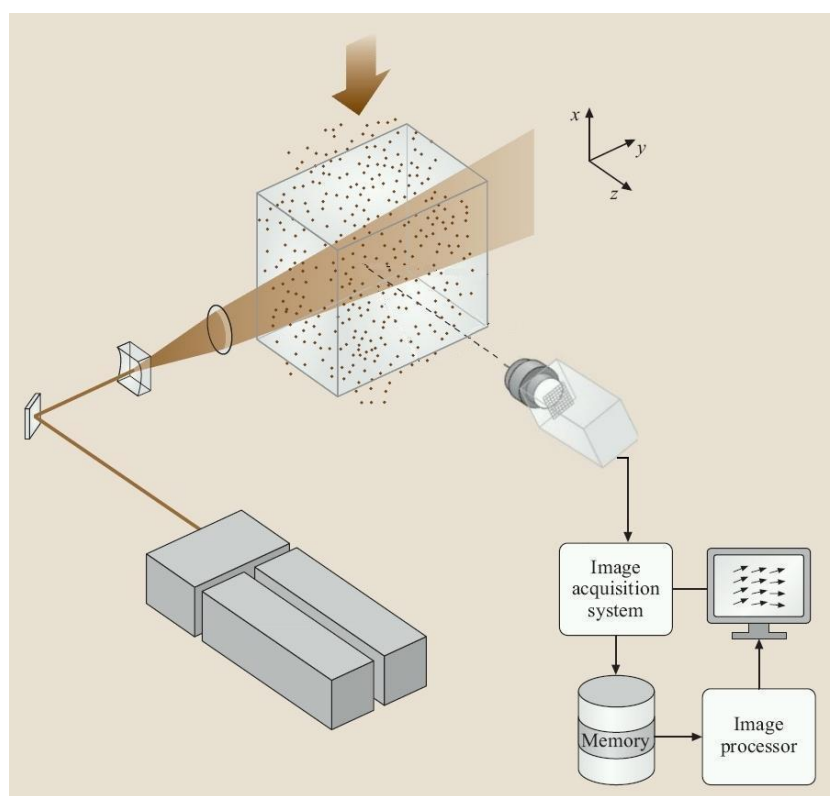

Figure 5: Schematic of a typical PIV measurement system

The main part of the experimental set-up (Fig. 5) consists of a transparent model of ribbed channel, laser light source (30 mJ double pulse Nd:YAG laser SoloPIV, New Wave Research, Inc.) and high resolution 12bit digital CCD camera $(1280 \times 1024$ pixels, PCO SensiCam). This system permits acquisition of two images at the minimum time interval of 200ns, exposition time of $5 \mathrm{~ns}$, and about $3.75 \mathrm{~Hz}$ repetition rate. The PIV recording system installed on $3 \mathrm{GHz}$ Pentium 4 computer with $3 \mathrm{~GB}$ RAM is capable for acquisition over 200 pairs of images during a single experimental run.

The PIV measurements were performed for pure air seeded with small droplets (few micrometers in diameter) of synthetic oil DEHS (Di-Ethyl-Heksyl-Sebacate). The oil drops volumetric concentration was very low $(<0.001)$, hence they did not affect the flow structure. 


\section{Experimental results and discussions}

\subsection{Experimental results - LCT}

In many cases, as mentioned above, remarkable enhancements of local and spatially averaged surface heat transfer rates are possible with rib turbulators, in spite of the low local Nusselt number at certain locations along the ribbed surfaces. Figure 2 shows the geometric details of the rib roughened test surface. Prior to this ribbed turbulator test section is a $255 \times 40 \mathrm{~mm}$ inlet duct that is $550 \mathrm{~mm}$ long. This is equivalent to 7.96 hydraulic diameters (where the hydraulic diameter is $69.11 \mathrm{~mm}$ ). The test surface that is analyzed contains a collection of rib turbulators that are perpendicular and angled with respect to the flow stream (Fig 3). To determine the surface heat flux (used to calculate heat transfer coefficients and Nusselt numbers), the convective power levels provided by the thermofoil heaters are divided by flat test surface area. Spatially resolved temperature distributions along the bottom rib turbulator test surface are determined using liquid crystals thermography (LCT) and true-colour image processing system commercially available from Data Translation Inc. [9].

In the discussion that follows, the Nusselt number is defined as:

$$
N u=h \cdot \frac{D_{h}}{k},
$$

where $\mathrm{h}$ is the heat transfer coefficient, $\mathrm{D}_{\mathrm{h}}$ is the channel hydraulic diameter and $\mathrm{k}$ is the molecular thermal conductivity of air.

The heat transfer coefficient is then based on the flat projected area and is determined using:

$$
h=\frac{q_{n}}{\left(T_{w}-T_{a}\right)}
$$

where $\mathrm{q}_{\mathrm{n}}$ is the local (net) surface heat flux, $\mathrm{T}_{\mathrm{w}}$ is a wall temperature detected by TLC and $\mathrm{T}_{\mathrm{a}}$ is the time - averaged, local mixed mean temperature of air.

The surface Nusselt number distribution along the rib roughened test surface and for centre of channel are presented in Fig 6 and Fig. 7 for four Reynolds number and geometry shown in Figs. 3a and 3c. It is evident that transverse vortex generators $\left(\mathrm{TVG}_{\mathrm{s}}\right)$ enhance heat transfer several times but only up to the certain Reynolds number. Comparison between the maps of the PIV velocity vectors and local Nusselt numbers contours reveals that above some limiting Reynolds number riblets does not enhance heat transfer. It seems that fluid at higher flow rates flowing over obstacles does not penetrate into the boundary layer and flows above the ribs. Hence, optimum flow condition should be established to find efficient enhancement of heat transfer augmentation in particular environment e.g. flow and heat exchanger configuration. 


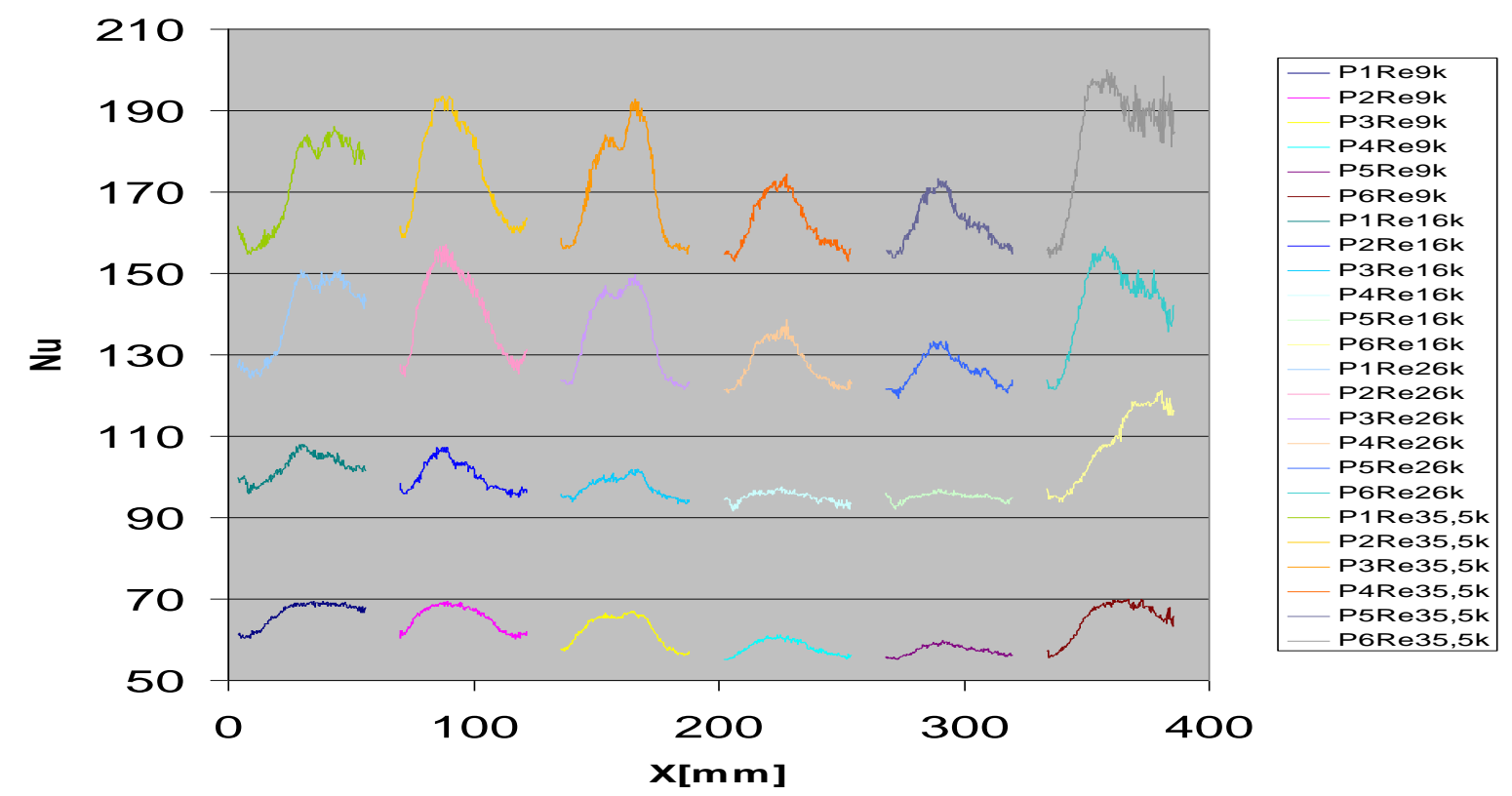

Figure 6: Nusselt number distribution in consecutive test section with a ribbed walls $(\mathrm{Re}=9000$, 16000,26000 and 35500). Six ribs are oriented perpendicular to the flow.

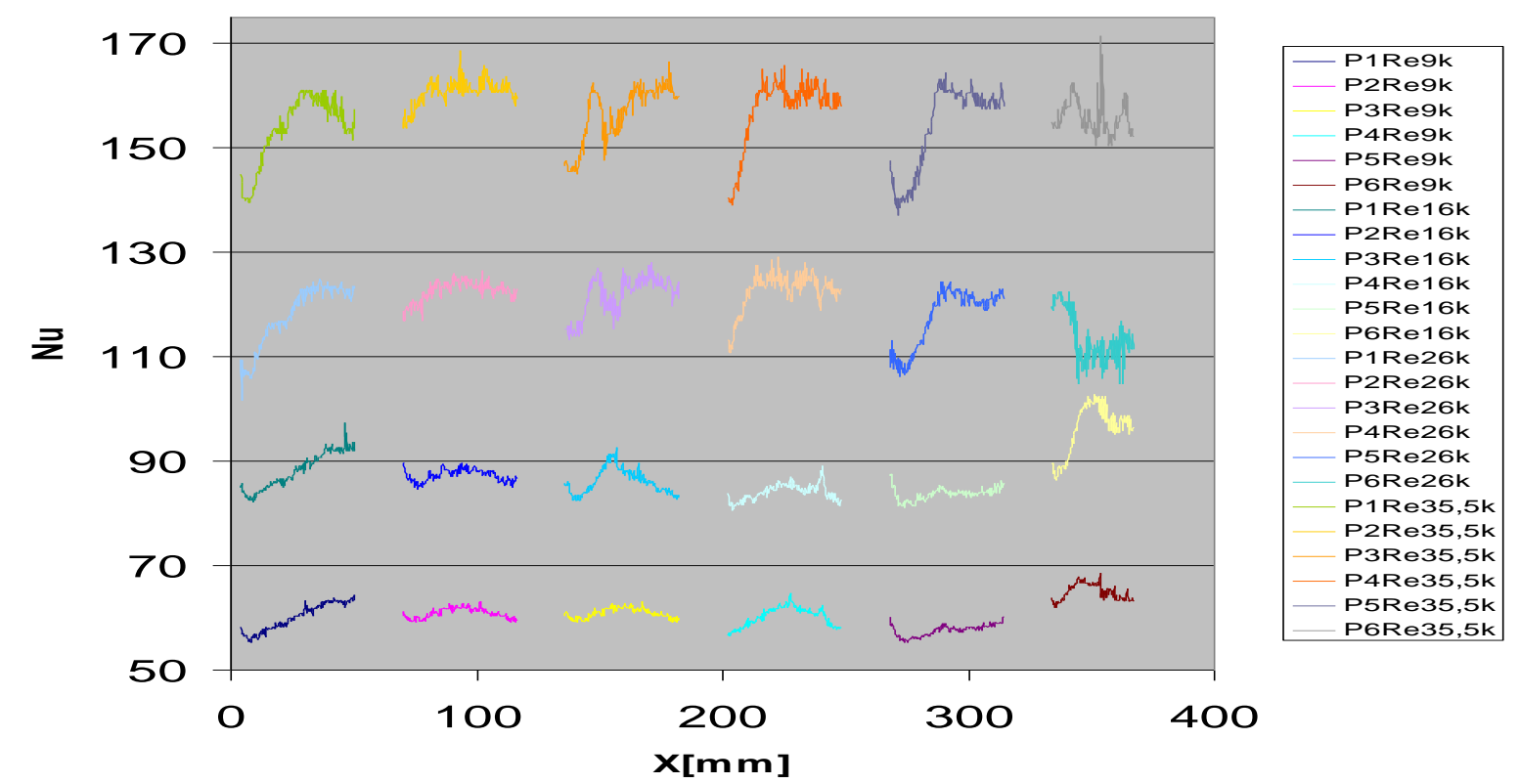

Figure 7: Nusselt number distribution in consecutive test section with a ribbed walls $(\operatorname{Re}=9000$, 16000,26000 and 35500). Six ribs are inclined at 45 deg to the flow.

For the purpose of comparison, preliminary tests for the baseline condition of a smooth channel (without ribs) with asymmetric heating were preformed. The local Nusselt number $\mathrm{Nu}$ was normalized by the Nusselt number for fully developed turbulent flow in smooth circular tubes given by the wall known Dittus-Boelter correlation:

$$
N u=0,023 \cdot \operatorname{Re}^{0,8} \cdot \operatorname{Pr}^{0,4}
$$

Nusselt number calculated according to Eq (6) for fully developed turbulent flow in smooth 


\section{K. Hanjalic et al.}

circular tubes and given Reynolds number e.g. 9000, 16000, 26000 and 35500 results in following data respectively: 29, 46, 68 and 87.

\subsection{Experimental results - PIV}

The PIV measurements were performed for 15 experimental configurations by collecting over 100 pairs of images for each case. Hence, 15 sets of velocity fields were obtained for 5 different ribs geometries and three Reynolds numbers ( $R e=9000,16000$ and 26000). The area interrogated by the PIV method was in all cases located in the mid-vertical-plane between side walls. Each set of measurements was used to evaluate mean velocity field and intensity of turbulence.
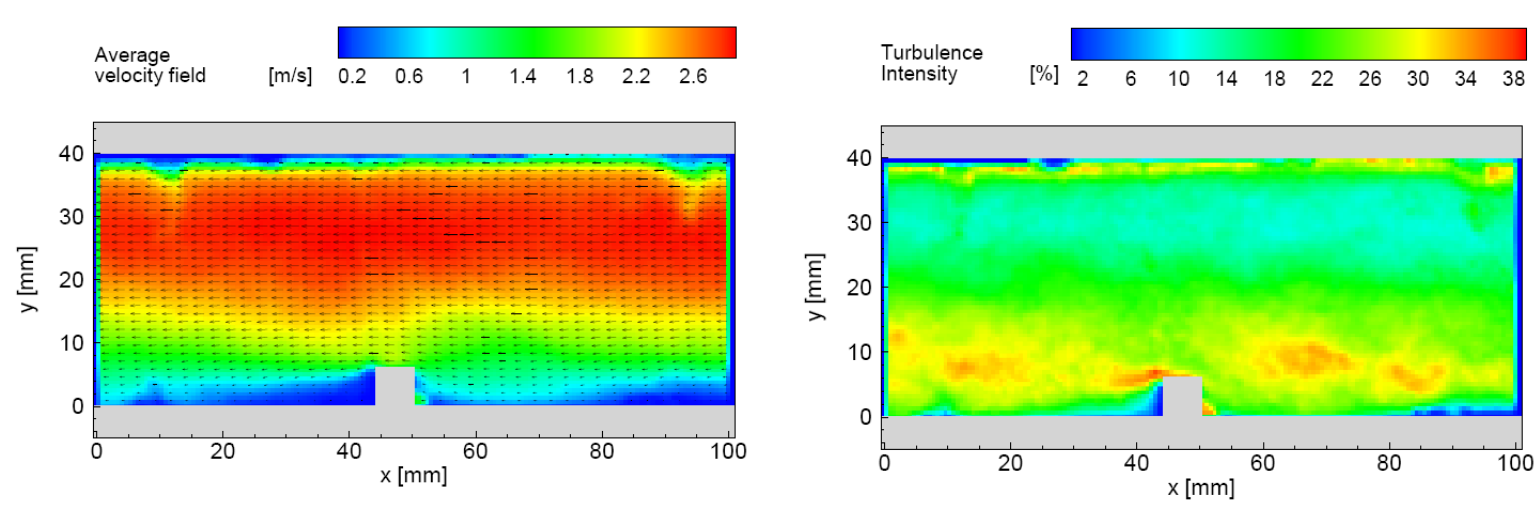

Figure 8: Averaged velocity field (left) and turbulence intensity (right) for geometry with ribbed bottom wall and ribs perpendicular to flow; Reynolds number $\operatorname{Re}=9000$.

For example Figure 8 left shows average velocity field (averaged over 100 instantaneous velocity fields) for geometry with ribbed bottom wall and the Reynolds number $\operatorname{Re}=9000$. Maximum velocity for this case is about $3 \mathrm{~m} / \mathrm{s}$, and is located in the top part of the channel. Presented averaged velocity field is computed from 100 instantaneous velocity fields, the turbulence intensity (Fig. 8 right) is defined as:

$$
I=\frac{\left(\frac{1}{n} \sum_{i=1}^{n}\left(u_{i}-\bar{u}\right)^{2}\right)^{\frac{1}{2}}}{\bar{u}} \cdot 100 \%,
$$

where $u_{i}$ and $\bar{u}$ are instantaneous and average velocity fields, respectively and $n$ is a number of measurements. Figure 8 (right) shows that flow is turbulent with maximum turbulence intensity about $38 \%$ in the vicinity of the rib and relatively small turbulence intensity in top part of the channel (about 10\%).

Figures 9 shows the same fields but for the Reynolds number $\mathrm{Re}=26000$. Maximum velocity is here about $8 \mathrm{~m} / \mathrm{s}$ and is located also in the top part of the channel. Turbulence intensity field, presented in Figure 9 right, shows that this intensity achieves maximum value 
(about $40 \%$ ) in the vicinity of the rib and decreases to $14 \%$ in top part of channel.

Figures 10 and 11 present similar results for the same Reynolds numbers but for inclined ribs as shown in Fig. 3c.
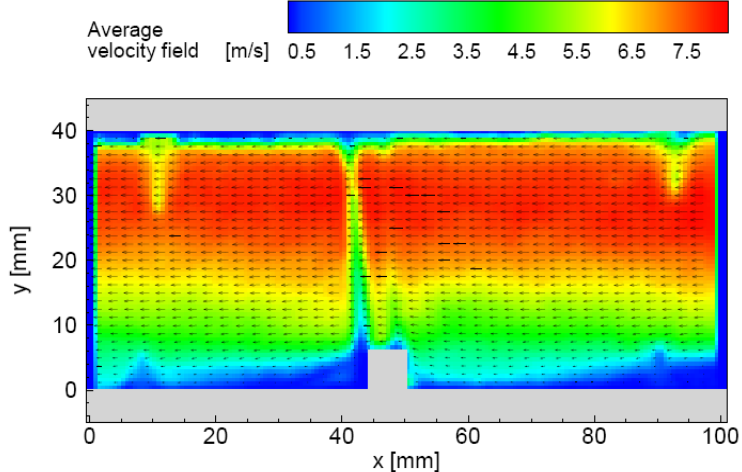

Turbulence Intensity
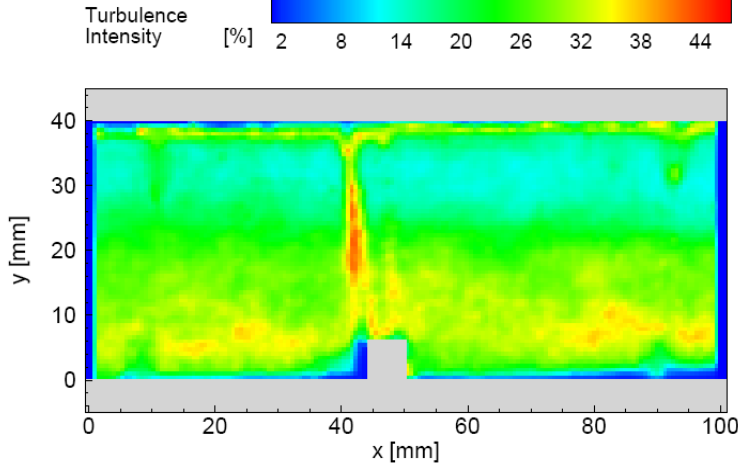

Figure 9: Averaged velocity field (left) and turbulence intensity (right) for geometry with ribbed bottom wall and ribs perpendicular to flow; Reynolds number $\operatorname{Re}=26000$.
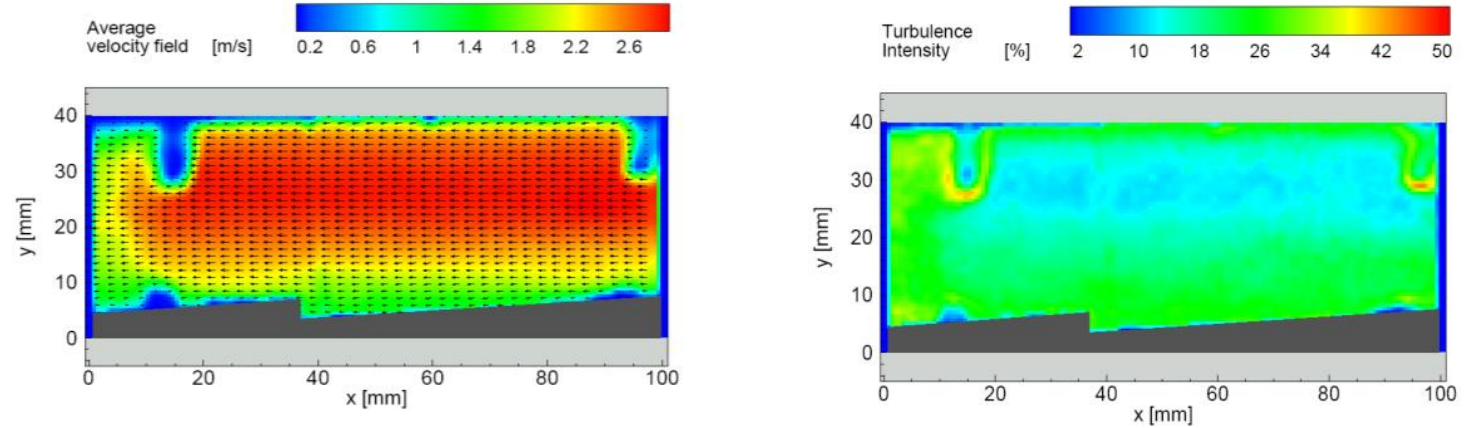

Figure 10: Averaged velocity field (left) and turbulence intensity (right) for geometry with ribbed bottom wall and ribs inclined at 45 deg to flow; Reynolds number $\mathrm{Re}=9000$.

Average
velocity

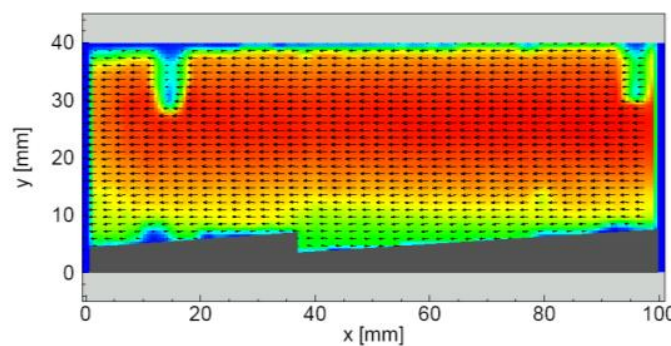

Turbulence Intensity

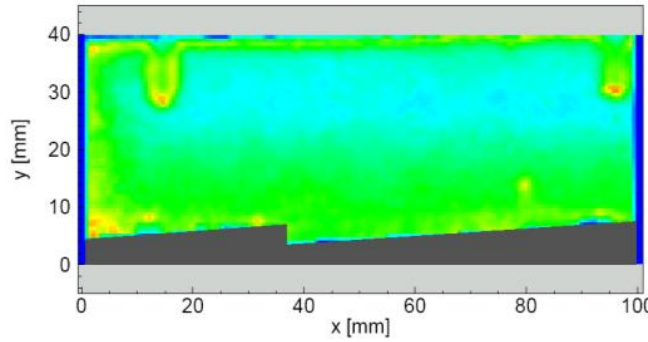

Figure 11: Averaged velocity field (left) and turbulence intensity (right) for geometry with ribbed bottom wall and ribs inclined at $45 \mathrm{deg}$ to flow; Reynolds number $\mathrm{Re}=26000$.

It should be mentioned that PIV images contain shadows produced by riblets and other obstacles (screws), visible in Figs. 9 - 11 as a large perturbations across the channel. These artefacts were excluded when the averaged turbulence was evaluated. 


\section{K. Hanjalic et al.}

\section{Conclusions}

New experimental techniques, in this case true-colour image processing of liquid crystal pattern and particle image velocimetry allow for new approaches to old problems and at the same time open up new areas of research. Image processed data make available quantitative, full-field information about the distribution of temperature, flow visualization and heat transfer coefficient, which will undoubtedly encourage the study of situation which have been, until now, too complex to consider. Here it was found that inclination of ribs leads to superior heat transfer performance because of the secondary flow induced by the rib angle. The corresponding average Nusselt number of the rib-roughened passages is generally two to three times higher in comparison with the smooth channel. Correlation of the flow velocity and heat transfer measurements elucidated mechanism limiting heat transfer coefficient for high flow rates. Future work will involve applying the present methods to more complex geometry and to unsteady flow.

\section{Acknowledgements}

The work reported in this paper includes scientific cooperation between University of Genoa, Italy, Center Mechanics, IPPT PAN, Warszawa, Poland and Gdansk University of Technology, Gdansk, Poland. The financial assistance of Ministry of Science and Higher Education, Poland, Grant No. 3T10B07329 is kindly acknowledged.

\section{References}

1. J.W. Baughn and Xiaojun Yan. Liquid Crystal Methods in Experimental Heat Transfer. Proc. $32^{\text {nd }}$ Heat Transfer and Fluid. California: Mechanics Institute Sacramento, pp 1540, 1991

2. M. Fiebig. Vortices: Tools to Influence Heat Transfer - Recent Developments, Proc. $2^{\text {nd }}$ European Thermal Sciences and $14^{\text {th }}$ UIT National Heat Transfer Conference. pp 41-56, 1996

3. M. Fiebig. Vortex Generators for Compact Heat Exchangers. J. of Enhanced Transfer Vol.2, pp 43-61, 1995

4. T. A. Kowalewski, P. Ligrani, A. Dreizler, C. Schultz and U. Fey in C. Tropea, J. Foss and A. Yarin (ed.), Handbook of Experimental Fluid Mechanic, Chap. 7, Springer-Verlag, Berlin, Heidelberg 2007

5. W. Leiner, K. Schulz, M. Behle and S. Lorenz. Imaging techniques to measure local heat and mass transfer. Proc. $3^{\text {rd }}$ I. Mech. E. Seminar Optical Methods and Data Processing in Heat and Fluid Flow. City University, London, 1-13 1996

6. R. J. Moffat. Experimental Heat Transfer Proc. The Ninth International Heat Transfer Conference, Jerusalem, Israel pp 187-205, 1990

7. M. Raffel, C. Willert, J. Kompenhans. Particle Image Velocimetry, A Practical Guide, Springer-Verlag, Berlin, 1998

8. J. Stasiek, A. Stasiek, M. Jewartowski and M. W. Collins. Liquid Crystal Thermography and True-Colour Digital Image Processing. Optics \& Laser Technology, Vol. 38, pp 243256, 2006

9. DATA TRANSLATION Ltd.: Image Processing Handbook, 1991

10. MERCK Ltd. Thermochromic Liquid Crystals Broom Road, Poole, U.K.

11. MINCO Products, Inc., Minnesota, USA 